\title{
VORLÄUFIGE MITTEILUNG ÜBER DIE BESCHAFFENHEIT DER OVARIALTASCHE VON CHRYSOCHLORIS, GALEOPITHECUS UND TUPAJA
}

\author{
VON \\ Dr. DANIEL DE LANGE Jr. \\ (UTRECHT).
}

Mit PI. VIII und IX

Im Jahre 1917 ist in den Anatomischen Heften die bekannte und schöne Arbeit Sobotta's erschienen über das Vorkommen und die Bedeutung der Ovarialtasche bei den Säugetieren '). Das Hauptergebniss dieser Arbeit ist, dass bei allen Säugetieren (vielleicht mit Ausnahne der Primaten und des Menschen) die Ovulation in einer geschlossenen Ovarialtasche stattfinde; dass das Ei im Anfang durch eine Erweiterung der Ampulle aufgesogen und mittels der Peristaltik der Tubamuskulatur durch den isthmischen Teil weiter nach dem Uterus, befördert werde. Die Flimmerung des Tubar- und Infundibularepithels scheine gar keine oder eine sehr geringe Rolle bei dem Eitransport zu spielen. Schon aus den Untersuchungen von ZUCKERKANDL (1897) und GERHARDT (1905) war bekannt, dass bei einigen Säugetieren (Muriden, Dipus, Sorex, Talpa, Mustelus, einigen Fledermäusen und Pinnipediern) die Ovarialtasche zeitlebens vollkommen gegen die Peritonealhöhle abgeschlossen ist, dass bei andren (Hund, Igel, Eichhörnchen und mehreren Fledermäusen) der Periovarialraum durch eine kleine Oeffnung mit der Peritonealhöhle verbunden ist, während ein dritte Gruppe (Huftieren, Kaninchen, Meerschweinchen und mehrere andere Säugetiere) eine weite Kommunikation zwischen diesen beiden Räumen aufweist, durch welche ein ansehnlicher Teil des Ovariums hervorsieht ${ }^{2}$ ).

Es ist der Verdienst Sobotta's nachgewiesen zu haben, dáss im letzterem Falle eine periodische Oeffnung und Schliessung der Ovarialtasche stattfindet: in der Weise, dass die Tasche in der Brunstund Ovulationsperiode geschlossen und ausserhalb derselben geöffnet sei. Die Möglichkeit der Taschenbildung hängt zusammen mit der Anwesenheit eines ventralen Gekröses, d. h. einer Ausbreitung des Mesosalpinx morphologisch ventralwärts von der Tuba. Dadurch ist zwischen den Schlingen des Oviduktes eine Mesenterialmembran ausgespannt, welche mittels eines, von der Infundibularregion nach der uterinen Längsmuskulatur verlaufenden Muskelbündels über das Ovarium hingezogen werden kann (Siehe Fig. 1). In einer späteren Auseinandersetzung dieses Problems betont Grosser, dass neben Muskelwirkung noch andre Momente beim Eitransport durch das Ovidukt im Spiele sein möchten. Er meint, Sobotta unterschätze die Wirkung der Flimmerung des Infundibular- und Ampullarepithels beim

1) I. SobotтA. Ueber den Mechanismus der Aufnahme der Eier der Säugetiere in den Eileiter und des Transportes durch diesen in den Uterus (Nach Untersuchungen an Nagetieren, Maus, Ratte, Kaninchen und Meerschweinchen). Anat. Hefte Bd. 54, H. 163, 1917.

2) Bei den Primaten und beim Menschen bildet die Ovarialtasche nur eine seichte Aushöhlung aus der der grössere Teil des Ovariums frei in der Bauchhöhle zu Tage tritt. Die Frage ob auch in diesem Falle die Möglichkeit eines zeitweiligen Verschlusses anwesend sei, lasse ich hier unberührt.

3) OtTo Grosser. Ovulation und Implantation und die Funktion der Tube beim Menschen. Arch. f. Gynäkol. Bd. 110 H. 2 1919. (Siehe auch Pflügers Arch. f. Physiol. Bd. 173). Für die vollständige Litteraturangabe siehe auch Sobotta (1917). 
Eitransport (zumal bei den Primaten) und ausserdem glaubt er, dass beim periodischen Verschluss der Ovarialtasche und bei der Erweiterung und Verengerung der Ampulle Erektionsvorgänge eine Rolle spielen dürften. Meine Befünde bei Galeopithecus unterstützen letztere Ansicht. Die Figur 1 stellt eine graphische Rekonstruktion der rechten Ovarialgegend von der ventro-medialen Seite gesehen dar. Die Tasche ist fast geschlossen; die Oeffnung derselbe ist als eine schmale, schief verlaufende Spalte unterhalb des Infundibulums zu ersehen. Letzteres is ganz nahe an den uterinen Abschnitt des Oviduktes gerückt. Der dicke, schwarze Strich $M$ stellt in schematischer Weise den Verlauf eines Bündels glatter Muskelfasern dar, welches von der einen Spitze der Spalte (in der Figur 1 rechts oben) zum uterinen Tuba-Abschnitt zieht und sich der Längsmuskulatur desselben anschliesst.

In der Figur 2 ist ein Schnitt durch diese Gegend abgebildet. Der Muskel wird mehr oder weniger in der Länge getroffen und strahlt an der inneren Seite des ventralen Gekröses aus ${ }^{1}$ ). Ich lenke die Aufmerksamheit auf die grossen Bluträume, welche sich; zumal an der oberen Seite der Figur, zwischen den Schlingen des Oviduktes vorfinden. Das Ovarium ist mehr oder weniger tangential geschnitten und enthält ein Corpus luteum. In einer der mittleren Schlingen der Tuba befindet sich ein Zellhaufen mit zum Teil degenerierten Kernen. Wahrscheinlich stellt derselbe den Rest eines Follikels dar, dessen Ei verloren gegangen ist, denn an einer andren Stelle ist ein Furchungsstadium von etwa 4 Zellen anwesend, von einem grossen Haufen dergleichen Zellen umgeben. An der äusseren Seite der Tubaschlingen verläuft ein Längsbündel glatter Muskelfasern welches auch in der Figur 3 sehr gut ersichtlich ist. In letzterer Figur ist an der inneren Seite ebenfalls ein Längsbündel zu beobachten. Einige Faserzügen verlaufen quer zwischen den grossen Bluträumen; das Zusammenziehen dieser Muskeln verursacht offenbar die Entleerung dieser Räume in das Sinussystem des Hilus, während die Längsbündel wohl bei der Eröffnung der Ovarialtasche eine Rolle spielen dürften. Eine gleiche Bedeutung lege ich zwei augenfälligen Muskelbündeln bei, welche sich an der Ampullarwand anheften und mehr oder weniger senkrecht $\mathrm{zu}$ den obengenannten verlaufen. In der Figur 4 habe ich einen dieser Muskel-komplexe abgebildet, dessen Fasern zum Teil an der Ampulle inserieren. Offenbar wird durch Kontraktion dieser Muskeln der Infundibularabschnitt der Tuba in der Richtung des Hilus zurückgezogen und dadurch die Ovarialtasche mehr oder weniger geöffnet. Eine Maximalöffnung wird auftreten bei Erschlaffung des Muskels $M$ und Zusammenarbeiten der beiden Längsmuskeln mit den beiden querverlaufenden.

Neben diesem muskulären Moment spielt aber wohl die geringere oder stärkere Füllung der grossen Bluträume der beiden Gekrösen eine wichtige Rolle. Ein Blick auf die Figuren 3 und 5 genügt, um einen Eindruck der Mächtigkeit dieses venösen Sinussystemes zu bekommen, welche es unwahrscheinlich macht, dass hier nur von einer nutritiven Funktion die Rede 'sei ${ }^{2}$ ). Allerdings finden sich die allergrössten Bluträume im Hilus, daneben kommen aber solche von grösser Machtigkeit im dorsalen und zum Teil auch im ventralen Tubengekröse vor. Diese venöse Bluträume hängen nach mehreren Seiten mit einander zusammen und bilden also ein spongiöses Sinussystem.

Während dieses venöse System stark entwickelt ist und daneben noch bedeutende Lymphräume vorkommen, treten die Arterien in Grösse, Anzahl und Verbreitung sehr zurück, wie ein Blick auf den Figuren 2-6 uns lehrt ${ }^{3}$ ). Ich erwartete daher eines unmittelbaren Uebergang von Arterien in diese venösen Räumen zu finden, wie das z. B. im Corpus cavernosum des Penis der Fall ist. Ich meinte etwas derartiges schon an der in der Figur 6 mit 3 angedeutete Stelle entdeckt zu haben, wo ein dünnwändiger, muskelarmer Sinus in ein dickes, muskulöses Gefäss übergeht, wie aus dem Vergleich

1) Die Spalte, welche den Muskel hart am Ovidukt durchquert ist ein Artefakt. Ein Vergleich mit der Figur 3 lehrt uns aber, dass die Spalte etwa der Ansatzstelle des ventralen Gekröses am Peritonealüberzug der Pars isthmica entspricht.

2) Sehr lehrreich ist der Vergleich mit den Bildern von Chrysochloris und Tupaja, wo doch die nutritive Funktion die gleiche bleibt und das venöse System der Gekrösen so schwach entwickelt ist, dass es bei schwacher Vergrösserung schwer aufzufinden ist.

3) Die Arterien sind mittels einer starken Kontur angegeben und jeder ist der Buchstabe a beigefügt. Ach hier ist der Vergleich mit den Bildern von Chrysochloris und Tupaja sehr lehrreich, weil da gerade das arterielle System verhältnissmässig viel besser entwickelt ist.

Wiewohl es mit dem hier behandelten in keinem Zusammenhang steht, möchte ich es nicht hinterlassen auf die starke Entwicklung des Urnierenrudiments und des Gärtnerschen Ganges hinzuweisen, welche zumal in der Figur 6 hervortritt. 
der Detailfiguren $6 a$ und $b$ sehr deutlich hervorgeht. Von diesen beiden Figuren (die dazu gehörenden Schnitten liegen 13 Schnitte also 120-130 $\mu$ aus einander) stellt die erstere zwei Sinus mit dünner Intima und fast ohne Muskelfasern dar, in der Figur $6 b$ sind die beiden Bluträume von einer starken Intima und von einer deutlichen Muskelhülle eingefasst. Unglücklicherweise zeigte aber keiner der beiden Sinus einen Zusammenhang mit dem arteriellen System. Das grössere, rechte Gefäss endete blind, das kleinere linke stand nach mehreren Seiten mit den Venensystem in Verbindung. Ich betrachte diese lokale Verdickung der Sinuswand als eine Art Stauvorrichtung, durch welche das Abfliessen des Blutes nach der Körperseite beeinträchtigt werden kann. Dadurch wird eine Erektion der Taschenwand bedingt, welche diese, ausserhalb der Brunstzeit gefalteten Membran vorstreckt. Durch Zusammenziehen des oben erwähnten Muskels $M$, welcher von der Infundibulargegend (quer zur Längsachse der Taschenöffnung) nach der Uteruswand verläuft, wird dann die Ovarialtasche noch weiter über das Ovarium hingezogen und die Oeffnung vollkommen abgeschlossen, damit die Ovulation im geschlossenen Periovarialraum stattfinden könne.

Wiewohl das venöse Sinussystem der beiden Gekrösen deutlich mit dem Kapillaren derselben zusammenhängt, erachte ich es wahrscheinlich, in Anbetracht der mehrfachen und innigen Verbindungen mit den grossen Bluträumen des Hilus, dass der Blutzufuhr hauptsächlich aus diesen letzteren stattfindet und dass einige dieser Verbindungen als zuführende, andere als abführende Wege von Blutstrom benutzt werden ').

Die Ovarialtasche von Chrysochloris zeigt uns in mancher Hinsicht ein ähnliches Bild, wie ein Blick auf den Figuren 7 und 9, zwei Rekonstruktionen der rechten bezw. der linken Ovarialgegend zweier verschiedener Individuen, uns lehrt. In beiden Fällen ist die Tasche nicht so ganz geschlossen, wie in der Figur 1 bei Galeopithecus. Ein kleiner Teil des Ovariums tritt aus der Tasche hervor. Es ist aber deutlich, dass eine weitere Verkürzung des Muskels $M$ den vollständigen Verschluss der Tasche verursachen werde. Die Figur 9 ist etwas mehr von der lateralen, die Figur 7 etwas mehr von der medialen Seite gesehen. Daher ist in ersterer der Teil des ventralen Tubagekröses, in dessen Rand der Schliessmuskel verläuft nach dem Beobachter gerichtet und weniger deutlich zu sehen als in letzterer.

In beiden Fällen ist die Konfiguration des Tubalschlingen nicht vollkommen die gleiche, aber das Verhältniss derselben zur Wand der Ovarialtasche d. h. zum dorsalen und ventralen Mesosalpinx und zum Ovarialhilus ist prinzipiell nicht verschieden. Das Verhältniss des Infundibulums zur Ovarialoberfläche ist aber wohl verschieden. Wie in der Rekonstruktion (Fig. 7) schematisch angedeutet ist und aus den Schnittbildern $7 a$ und $7 b$ hervorgeht befindet sich bei diesem Objekt ein bedeutender Zipfel des Infundibulums ausserhalb des Ovarialraumes; während das Ovarium an der lateralen Seite teilweise von einem anderen Zipfel desselben umfasst wird (siehe zumal die Figur $7 b$ ). Beim andren Objekt setzt sich das hohe Infundibularepithel wohl noch eine Strecke auf die äussere Seite der Taschenwand fort, aber vom einem freien, hervortretenden Zipfel ist keine Rede. Der mediale, äussere Zipfel befindet sich hier innerhalb der Tasche, und daher ist das Ovarium allseitig vom Infundibularepithel eingefasst, wie aus den Figuren $9 a$ und $b$ hervorgeht. Auch ohne volkommenen Verschluss der Ovarialtasche ist also eine Beförderung der reifen Eier ausserhalb derselben äusserst unwahrscheinlich. Nur Eier aus ganz an der kaudalen Seite liegenden Follikeln können bei unvolkommenem Verschluss in die Peritonealhöhle gelangen, weil sonst allentwegen das Infundibulum sich der Oberfläche des Ovariums angeschmiegt hat (siehe die Fig. $9 a$ und $b$ ). Dass aber die Ovarialtasche weit geöffnet sein kann, beweisen die Verhältnisse der anderseitigen Taschen derselben Objekten, wo die Wand der Tasche sich in Falten gelegt hat und der grössere Teil der Ovarialoberfläche frei zu Tage tritt, wie man aus der Figur 8 ersehen kann ${ }^{2}$ ).

Wiewohl diese Sachlage vielleicht beim Herausschneiden des Urogenitalapparates entstanden sein

1) Klappenvorrichtungen, welche Fingerzeige nach der Richtung des Blutstromes geben würden, habe ich nicht entdeckt.

2) Ich lenke die Aufmerksamheit auf die Tatsache, dass in den Falten des Mesosalpinx, aber ausserhalb der Region des Infundibularepithels ein vier- bis sechs-zelliger Furchungskugel anwesend ist. Diese sonderbare Lage ist wohl artifiziell und beim Herauspräparieren entstanden. 
möge '), beweist dieser Umstand, dass das Ovarium leicht aus der Tasche hervortreten kann und dass wir hier ein Beispiel der dritte Gruppe vor uns haben, deren Mitglieder eine periodische Alternation zwischen Verschluss der Ovarialtasche und Hervortreten des Ovariums aufweisen.

Was für Bedeutung die Tatsache besitzt, dass bei Chrysochloris in allen 4 untersuchten Fällen die beiderseitigen Ovarialtaschen des gleichen Individuums einen gegensätzlichen Zustand (also Eröffnung bezw. Verschluss der Tasche) aufweisen, ist mir nicht klar geworden. Man sollte doch nach den musterhaften Untersuchungen Sobotta's erwarten, dass die beiden Seiten eines Individuums sich in Anbetracht der Brunst in der gleichen Periode befinden.

Was nun den Mechanismus der Eröffnung bezw. des Verschlusses der Ovarialtasche anbetrifft so genügt, ein Blick auf die Schnittbilder der beiden Serien im Vergleich mit denen von Galeopithecus um uns zu überzeugen, dass hier von einem bedeutenden Einfluss der Blutstauung keine Rede sein kann. Grössere Bluträume treten in den Tuba-Gekrösen nirgend auf. Kleinere Arterien und Venen sind ziemlich gleichmässig in denselben verbreitet, zum grösseren Teil sind sie aber zu klein, um in den Figuren mit eingezeichnet werden zu können. Nur in der Marksubstanz und im Hilus des Ovariums kommen grössere Spalten und Räume vor, welche aber teilweise als Lymphräume zu betrachten sind. Jedenfalls sind auch letztere so spärlich in der Taschenwand vertreten, dass sie für irgend einen Erektionsmechanismus von keiner Bedeutung sein können. Eröffnung und Schliessung können also nur auf Muskelwirkung beruhen. Die Lage des Schliessmuskels habe ich schon angegeben; dieselbe stimmt mit den Befunden bei andren Tieren. Für die Eröffnung kommt die Längsmuskulator der Tuba in Betracht, sowie Muskelzüge, die vom Hilus in die Taschenwand ausstrahlen (siehe die Figg. $7 a$ und $9 a$ und $b \mathrm{M}^{\prime}$ ).

Bei Tupaja ist die Sachlage eine andere. Offenbar zeigt die Ovarialtasche dieses Tieres den zweiten Typus: d.h. eine offene Verbindung des Ovarialraumes mit der Peritonealhöhle ist anwesend, aber die Beschaffenheit derselben ist derartig, dass von einem Hervortreten des Ovariums ausserhalb der Tasche keine Rede ist. Diese Erscheinung wird in diesem Falle nicht nur durch die Enge der Oeffnung sondern hauptsächlich durch die starke Einrollung der Tuba bedingt. Die Pars isthmica und das Infundibulum ueberkreuzen sich. Letzteres liegt nicht dem Uterus gegenüber sondern demselben angeschmiegt und ist teilweise mit demselben verwachsen. Denkt man sich in der Rekonstruction der Ovarialgegend von Chrysochloris (Fig. 7) das Ovarium als eine Kugel, dann könnte man sagen, dass bei diesem Tiere die Tuba etwa $270^{\circ}$ der Ovarialoberfläche umfasst, während bei Tupaja das Infundibulum 90 bis $120^{\circ}$ weiter vorgerückt ist, also die ganze Oberfläche oder etwas mehr umschlungen hält. Dadurch ist der isthmische Abschnitt des ventralen Gekröses über das Infundibulum hinweggezogen, wie man aus der Figur 10 ersehen kann.

Dieser Schnitt ist etwa quer zur Längsachse des Uterus geführt und trifft eben die blindsackartige Kuppe derselben. Oben ist die laterale, unten die mediale Seite. Die Falten des Infundibulums sind teilweise mit dem Peritonealüberzug der Gebärmutter verwachsen, teilweise ragen sie frei in dem vom ventralen und dorsalen Tuba-Gekröse begrenzten Periovarialraum. Sie treten aber nicht durch die spaltförmige Oeffnung zwischen ventralen Tuba-Gekröse und Uteruswand in der Peritonealhöhle hervor.

Die Möglichkeit, dass ein im Periovarialraum freikommendes Ei in die Peritonealhöhle gelange, ist äusserst klein, denn wie aus der Figur 10 hervorgeht, hätte dasselbe dazu zuerst die Spalten zwischen den Infundibularfalten und später noch die eigentliche schmale Oeffnung der Tasche zwischen ventralen Gekröse und Gebärmutter zu passieren. Diese Oeffnung kann mittels einer sehr einfachen Muskelvorrichtung geschlossen werden, weil das ventrale Tubagekröse etwas mehr distalwärts mit dem Peritonealüberzug des Uterus verwachst und dann den Anfang des uterinen Abschnittes des Oviduktes enthält. Dies geht aus der Figur 11 hervor, in welcher die Tuba aus der Uterusschleimhaut hervorwächst und die Ovarialtasche vollkommen geschlossen ist. In den mehr proximalen Schnitten, so zusagen oberhalb der Ueberkreuzung der Infundibularregion durch die Pars isthmica tubae und deren Gekröse, überragt eine feine Spalte des Infundibulums (der Rest der in der Figur 10 mit einem

1) Ich verfüge nicht über die ganzen Tieren sondern nur über die auspräparierten Urogenitalapparaten, welche in Hinsicht auf mögliche Schwangerschaft für das Embryol. Institut gesammelt wurden. 
Kreuz bezeichnete Oeffnung) den Rand des ventralen Gekröses um 50 bis $60 \mu$. Durch dieselbe ragt ein Zipfel des Infundibulums in der Peritonealhöhle hinein, wie aus der Figur 12 hervorgeht, wo der Ovarialraum schon wieder verschlossen ist (Das Kreuz zeigt wieder die Stelle der Spalte in mehr distalen Schnitten an). Weil aber die obengenannte feine Spalte hart am Peritonealüberzug der Gebärmutterkuppe liegt, bedarf es nur eine ganz kleinen Verschiebung der Ovarialkomplexes um die Oeffnung vollkommen abzuschliessen. In der Figur 12 ist der Uterus nicht ganz miteingezeichnet, weil nur die Muskelhülle der Gebärmutterkuppe gestreift und die Schleimhaut schon aus dem Schnittbild verschwunden ist. Bei dieser Sachlage würde ein Hervortreten des Ovariums aus der Tasche sehr schwierig sein. Diese Erscheinung wurde daher in den von mir bearbeiteten Serien niemals beobachtet. Auch fehlt ein ausgebildeter Eröffnungs-bezw. Schliessungsmechanismus. Grosse Bluträume wird man vergebens in der dünnen Taschenwand suchen, auch Muskeln kommen nur an der uterinen Seite im Gekröse vor und können also nur Bedeutung haben für die Erweiterung der schmale Spalte zwischen letzterem und der Gebärmutter. Man könnte sich denken, dass die Längsmuskulatur der Tuba eine Rolle spiele beim Ausrollen des Oviduktes, aber auch das ist ausgeschlossen, weil das Infundibulum mit dem Peritonealüberzug der Uteruskuppe verwachsen ist (siehe die Figg. 10 und 12). Summa Summarum ist bei Tupaja also nur eine zeitweilige kleine Erweiterung der Oeffnung zwischen Periovarialraum und Peritonealhöhle möglich, aber ein periodisches Hervortreten des Ovariums ausserhalb der Ovarialtasche ist ausgeschlossen.

\section{FIGUREN-ERKLÄRUNG.}

\section{ALLGEMEINGÜLTIGE ZEICHENERKLÄRUNG.}

$$
\begin{aligned}
\mathrm{a} & =\text { Arteria } \\
\mathrm{A} & =\text { Pars ampullaris Tubae } \\
\mathrm{C} & =\text { Corpus luteum } \\
\mathrm{Dk} & =\text { dorsales Tuba-Gekröse } \\
\mathrm{E} & =\text { sich furchendes Ei } \\
\mathrm{F} & =\text { Fettgewebe im Hilus } \\
\mathrm{Fo} & =\text { Graaffsche Follikel } \\
\mathrm{G} & =\text { Gärtnerscher Gang } \\
\mathrm{Hi} & =\text { Hilus }
\end{aligned}
$$

$$
\begin{aligned}
\mathrm{Ho} & =\text { Ovarialhilus } \\
\mathrm{I} & =\text { Infundibulum } \\
\mathrm{Ist} & =\text { Pars isthmica Tubae } \\
M & =\text { Schliessmuskulatur der } \\
& \text { Ovarialtasche } \\
M^{\prime}= & \text { Eröffinungsmuskulatur der- } \\
& \text { selben } \\
\mathrm{cM} & =\text { Circulärmuskulatur des } \\
& \text { Uterus }
\end{aligned}
$$

$$
\begin{aligned}
& 1 M=\text { Längsmuskulatur derselben } \\
& \mathrm{Ov}=\text { Ovarium } \\
& \mathrm{Po}=\text { Periovarialraum } \\
& \mathrm{Sp}=\text { Spalte der Ovarialtasche } \\
& \mathrm{U}=\text { Urnierrudiment } \\
& \mathrm{Ut}=\text { Uterusschleimhaut } \\
& \mathrm{Utl}=\text { Uteruslumen } \\
& \mathrm{Vk}=\text { ventrales Tuba-Gekröse }
\end{aligned}
$$

In den Schnittbildern sind die Arterien mittels einer starken, die Lymphräume mittels einer schwachen Kontur angedeutet, die Venen und die venösen Sinus sind schwarz eingefüllt.

\section{Galeopithecus.}

Fig. 1. Graphische Rekonstruktion der rechten Ovarialgegend von Galeopithecus. $\times 25(2 / 3)$.

Die Ovarialtasche wird von der medioventralen Seite her gesehen; die Taschenwand sowie alle Mesenterialbildungen sind punktiert und wie durchsichtig dargestellt. Innerhalb derselben sind das flach schraffierte Ovarium und die körperlich dargestellte Tuba zu ersehen. Diese beiden Organen sind als undurchsichtig betrachtet. An der abgewendeten Seite der Figur ist die Grenze des Hilus mittels Kreuzen und ist die Ansatzstelle des Ovariums mittels Kreuzen und Pünkte angedeutet worden. Die numerierten Linien geben die Lage der Schnittbilder an.

Fig. 2. Schnitt durch den medialen Abschnitt der Ovarialtasche um den Verlauf des Schliessmuskels $M$ zu demonstrieren. Die spaltförmige Oeffnung der Ovarialtasche ist nicht getroffen. $\times 25(2 / 3)$.

Fig. 3. Schnitt durch die mittlere Region der Ovarialtasche zur Demonstration der grossen Bluträume im dorsalen Gekröse. Die Taschenöffnung ist getroffen, einerseits wird dieselbe von Infundibularepithel begrenzt. $\times 25(2 / 3)$.

Fig. 4. Schnitt durch den lateralen Abschnitt der Tasche. Die Ampullarwand und das Ovarium sind gerade tangential getroffen. Zahlreiche Schläuche des Urnierenrudiments (U) sowie querverlaufende Muskelzüge $\left(M^{\prime}\right)$ sind zu beobachten. $\times 25(\% / 3)$.

Fig. 5. Ein anderer Schnitt durch die mittlere Region der Tasche. Infundibulum und Spalte sind in der Mitte getroffen. Man beachte die grossen Bluträume im Hilus, im Ovarium und in den beiden Tuba-Gekrösen. $\times 35(1 / 2)$.

Fig. 6. Ein anderer Schnitt durch den lateralen Abschnitt der Tasche. Die Ampulle ist der Länge nach getroffen. Màn beachte die mit 3 angedeuteten Bluträume und das ansehnliche Rudiment des Gärtnerschen Ganges und der Uniere. $\times 35(1 / 2)$.

Fig. $6 a$ und $b$. Detailfiguren der in der vorigen Figur mit 3 angedeuten Bluträume. In der Figur $6 a$ sind dieselben dünnwändig und ohne deutliche Muskeln; in der Figur $6 b$ sind die Wände dick und muskulös. $\times 250\left({ }^{2} / 3\right)$. 
Chrysochloris.

Fig. 7. Graphische Rekonstruktion der rechten Ovarialgegend eines Exemplars von Chrysochloris. $\times 35(2 / 3)$. Das weisse Feld stellt den nach aussen zurückgeschlagenen medianen Zipfel des Infundibulums dar. Für die weitere Erklärung siehe Fig.' 1 .

Fig. 7a. Schnitt des gleichen Objektes etwa senkrecht zur Längsachse der Tasche und der Gebärmutter. $\quad \times 70(1 / 9)$

Die Oeffnung der Tasche ist gerade noch nicht getroffen; die Stelle wo dieselbe wenige Schnitte weiter auftritt ist mittels zweier Kreuzen angedeutet. Man beachte die Schliessmuskulatur $M$ und die Eröffnungsmuskeln $M^{1}$.

Fig. 7b. Schnitt durch die Taschenöffnung des gleichen Objektes. Man beachte den Uebergang des Infundibulums in der Ampulle und den Zipfel des ersteren, welcher das Ovarium an der lateralen Seite einhüllt. $\times 35(1 / 1)$.

Fig. 8. Schnitt durch die linke Ovarialregion des gleichen Individuum. Der Schnitt ist etwa parallel der Längsachse der Gebärmutter geführt, also senkrecht zur Schnittführung der Serie der rechten Seite. Man beachte die starke Faltung des Infundibulums und der Taschenwand, die ganz freie Lage des Ovariums, die Anwesenheit eines sich furchendes Ei $(E)$ zwischen den Falten des Infundibulums. $\times 70(1 / 2)$.

Fig. 9. Graphische Rekonstruktion der linken Ovarialgegend eines anderen Individuums von Chrysochloris. $\times 35(2 / 3)$. Für die weitere Erklärung siehe Fig. 1.

Fig. 9a. Schnitt durch den oberen, geschlossenen Taschenabschnitt. Die Zipfel des Infundibulums hüllen das Ovarium vollständig ein. $\times 50(2 / 3)$.

Fig. 9b. Schnitt durch den medialen Teil der Taschenöffnung. An der laterale Seite ist die Oeffnung noch nicht getroffen. Der Schnitt hat den Ovarialhilus getroffen. $\times 50(2 / 3)$

Tupaja.

Fig. 10. Schnitt durch die Oeffnung der rechten Ovarialtasche von Tupaja. Schnittführung senkrecht zur Längsachse der Gebärmutter. Die Uterusschleimhaut ist gerade noch gestreift. Das Infundibulum ist mit dem Uterusperitoneum verwachsen und wird vollständig von dem ventralen Tuba-Gekröse überdeckt. Die obere Seite der Figuren ist die laterale, die untere ist die mediale. $\times 40(1 / 2)$.

Fig. 11. Etwas mehr distaler Schnitt des gleichen Objekts. Die Abgangsstelle der Tuba von der Uterusschleimhaut und das Uteruslumen sind $\mathrm{zu}$ ersehen. Die. Ovarialtasche ist geschlossen und das Infundibulum ist aus dem Schnittbild verschwunden. $\times 40(1 / 2)$.

Fig. 12. Etwas mehr proximaler Schnitt des gleichen Objektes. Das ventrale Tuba-Gekröse ist mit dem Infundibulum verwachsen. Ein Zipfel des letzteren ragt in der Peritonealhöhle hinein. Die Stelle der Spalte des Infundibulums in den mehr distalen Schnitten ist mittels eines Kreuzes angedeutet. $\times 40(1 / 2)$. 


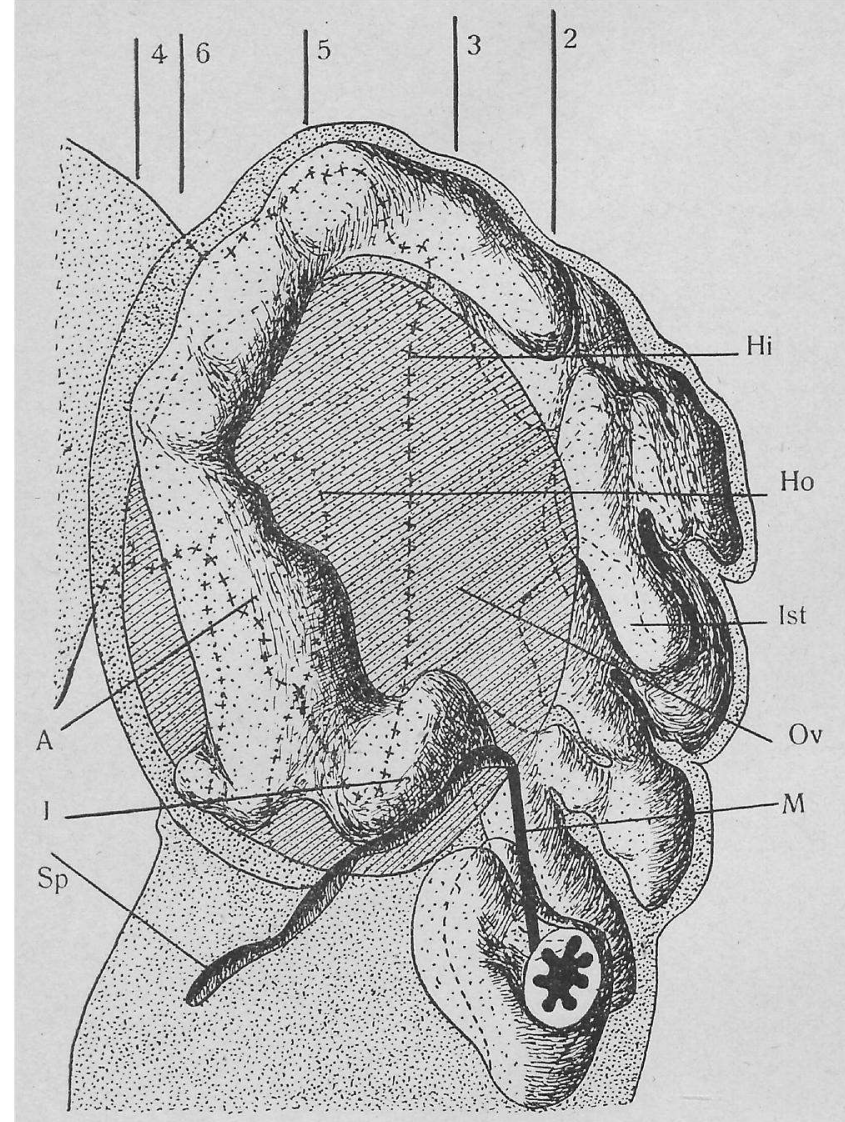

Fig. 1.

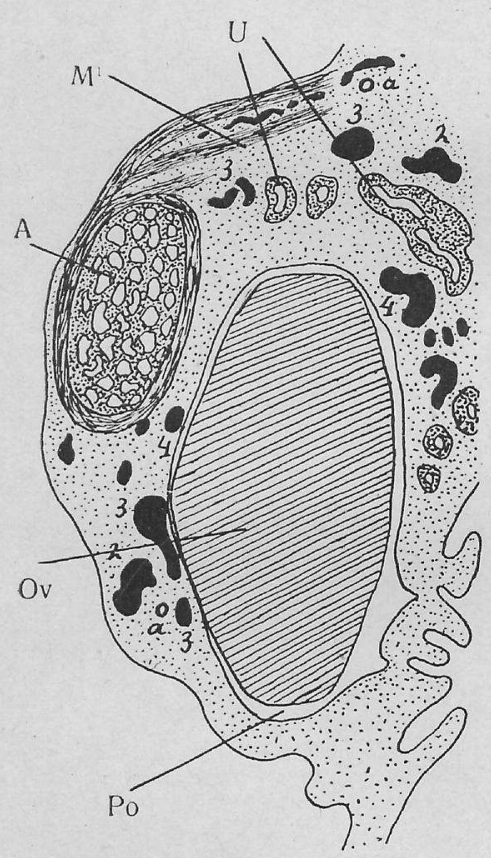

Fig. 4.

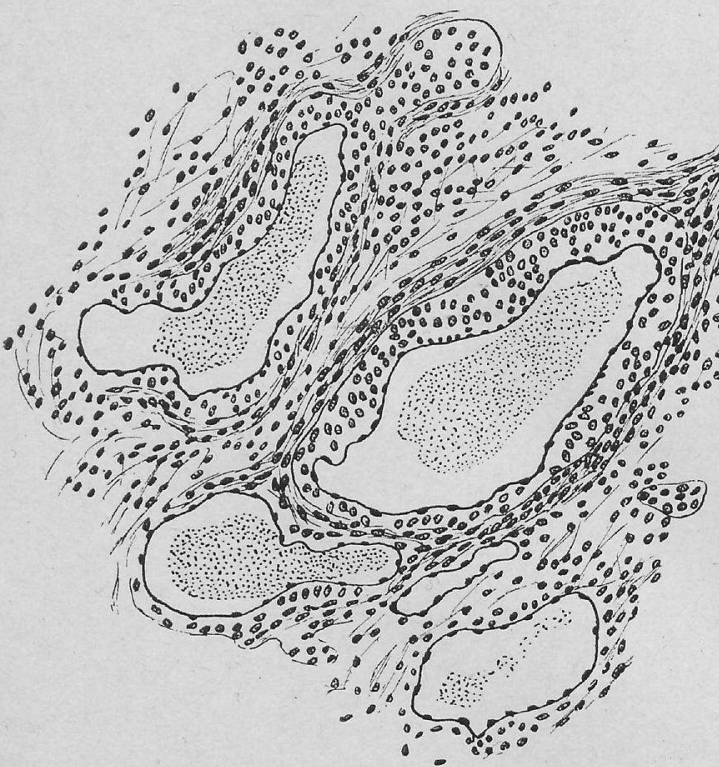

Fig. $6 b$.
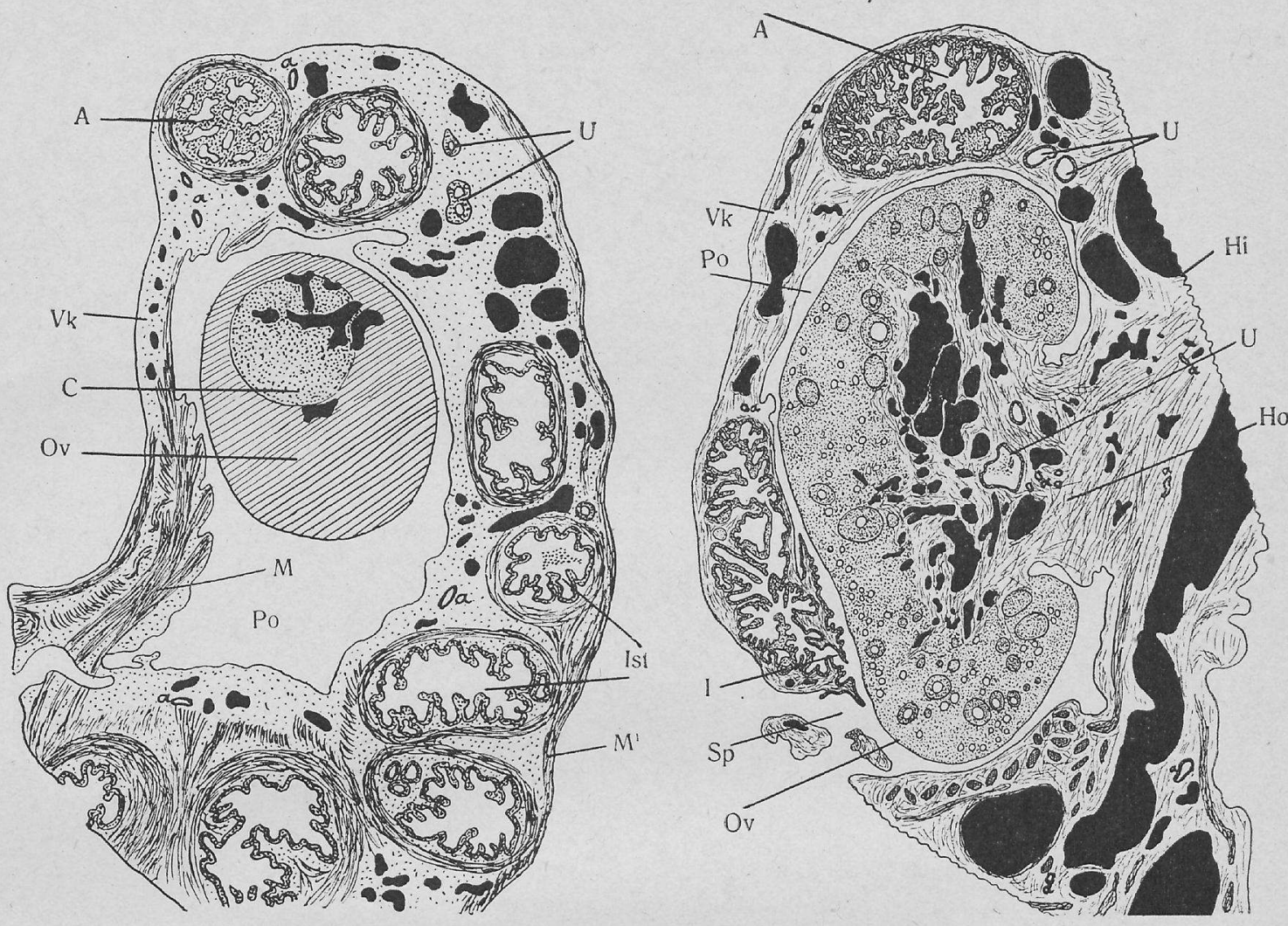

Fig. 5 .
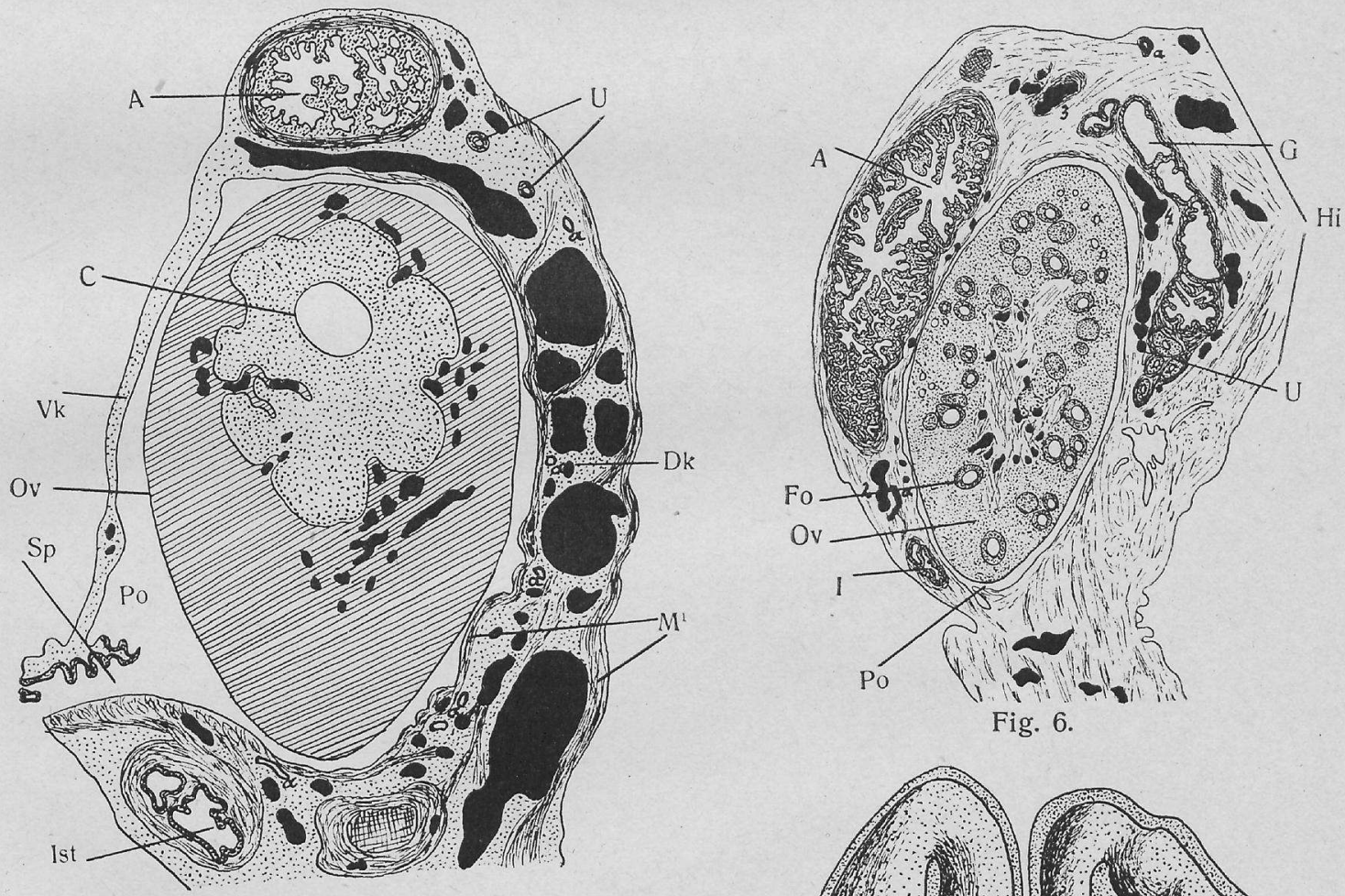

Fig. 6.

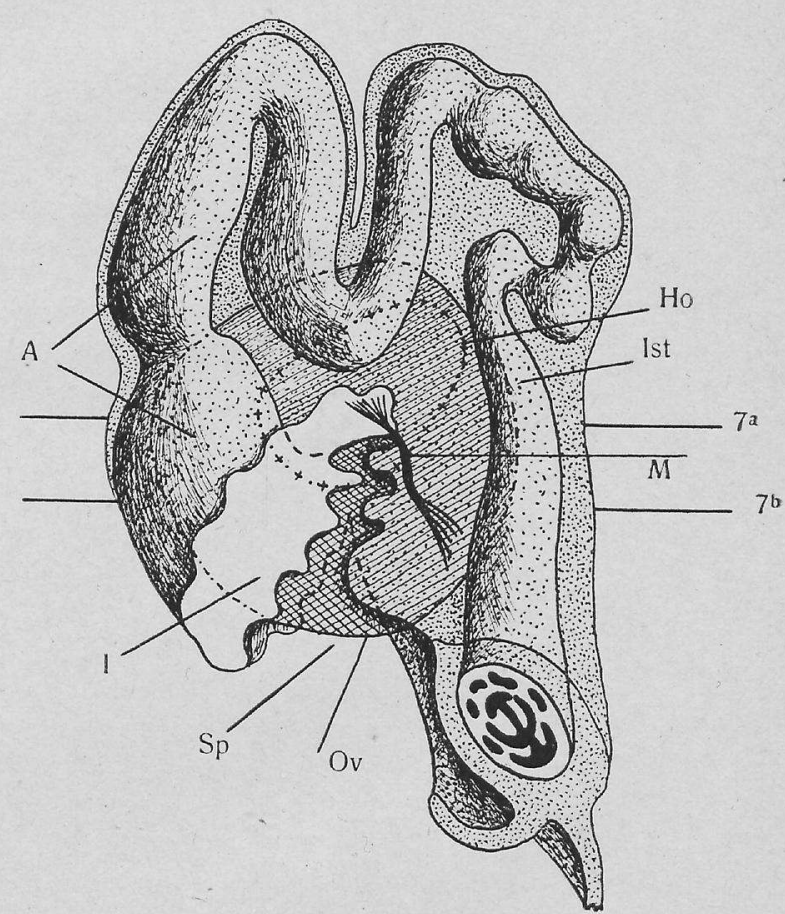

Fig. 7. 


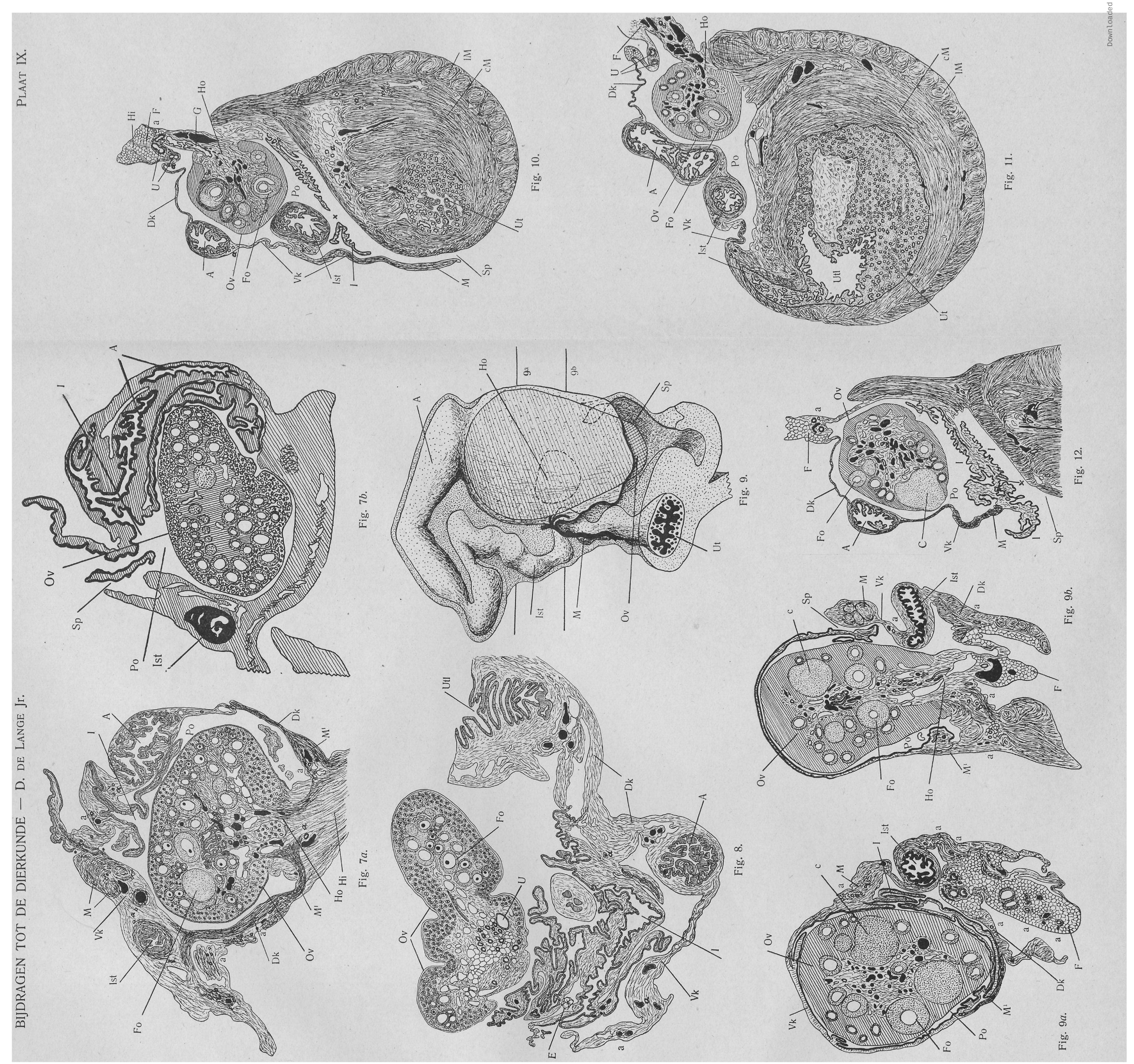

